\title{
Comparative Approach for Hyper-pigmented Gingiva
}

\author{
Jaishree Garg ${ }^{1}$, Rajkumar Maurya ${ }^{2}$, Ankur Gupta $^{3}$, Harpreet Singh ${ }^{4}$, Harsh Ashok Mishra ${ }^{5}$
}

${ }^{1}$ Department of Periodontics, I.D.S Barelly; ${ }^{2}$ Department of Orthodontics, Army Dental Center (R \& R), Delhi; ${ }^{3}$ Department of Orthodontics, Institute of Dental Sciences, Bareily; ${ }^{4}$ ESI Dental College \& Hospital, New Delhi; ${ }^{5}$ Late Shri Yashwantrao Chavan Memorial Medical \& Rural Foundation Dental College, Ahmednagar, Maharashtra, India.

\section{Corresponding Author: \\ Dr. Jaishree Garg \\ Email: bracedbyraj@gmail.com}

This is an Open Access article distributed under the terms of the Creative Commons Attribution License (creativecommons.org/ licenses/by/3.0).

Received

Accepted

Published

October 25, 2016

January 19,2017

February 10, 2017

\begin{abstract}
Melanin pigmentation is known to be caused by melanin granules within the gingival epithelium. Excessive gingival pigmentation is a major esthetic concern for many people. Though, it is not a medical problem, many people complain of dark gums as unesthetic. The degree of pigmentation varies from person to person. Although clinical melanin pigmentation does not present a medical problem, demand for cosmetic therapy is commonly made by people with moderate gingival melanin pigmentation. This case report presents a new surgical technique of de-epithelization which has been successfully used to treat gingival hyperpigmentation caused by excessive melanin deposition and highlights the relevance of an esthetically pleasing smile specially in smile conscious individuals.
\end{abstract}

Keywords: Esthetics, Gingiva, Hyperpigmentation, Melanins, Pigmentation.

\section{Introduction}

Melanin pigmentation of the gingiva occurs in all races [1]. Melanin, a brown pigment, is the most common natural pigment contributing to endogenous pigmentation of gingiva and the gingiva is also the most predominant site of pigmentation on the mucosa. Melanin pigmentation is the result of melanin granules produced by melanoblasts intertwined between epithelial cells at the basal layer of gingival epithelium [2]. Melanin pigmentation of gingiva is symmetric, persistent, does not alter normal gingival architecture occuring as a result of genetically determined hyperactivity of melanocytes. Melanocytes of dark skinned and black individuals are uniformly highly reactive than in light skinned individuals. Although clinically melanin pigmentation of the gingiva does not present any medical problems it can be an esthetic concern for the patient [3].

Various depigmentation techniques have been employed with similar results such as chemicals, abrasion with diamond burs, gingivectomy, soft tissue autografts, partial thickness flap, cryosurgery and lasers. Each technique has its own advantages and disadvantages [4]. The de-epithelialization essentially involves surgical removal of gingival epithelium along with a layer of the underlying connective tissue and allowing the denuded connective tissue to heal by secondary intention, and which is devoid of melanin pigmentation. The present case reports, describe a simple and effective surgical depigmentation technique that does not require sophisticated instruments or apparatus yet yields esthetically acceptable results along with patient's satisfaction.

\section{Case Report}

A 27 year old male patient and a 19 year old male visited the department of Periodontics for routine oral prophylaxis. On intraoral examination, diffused blackish pigmentation of gingiva was seen in both the cases [Fig.1a,1b]. The unsightly 
gingival pigmentation was pointed out to the patients and they were made aware about the array of aesthetic treatment options available. The patients had also noticed the gingival pigmentation since a year and of their own accord opted to undergo the depigmentation procedure. Depigmentation procedures were planned after obtaining patients consent. The patients were given oral hygiene instructions and they underwent oral prophylaxis in phase I periodontal therapy. The procedure was carried out from premolar to premolar region in the first patient and canine to canine in the other patient in the maxillary anterior region for both the cases [Fig.2a,2b] using deepithelialization technique after adequate local anesthesia. The uppermost layer of the gingiva was carefully scraped using 15 number blade which was held parallel to the long axis of the teeth. The hyperpigmented epithelium was removed [Fig.3], minimum of force/pressure was used to avoid postoperative gingival pitting. Bleeding was controlled with a sterile gauze pressure pack. Surgical areas were covered with a periodontal pack [Fig.4] and post-operative instructions were given. In second patient, de-epithelialization was carried out by bur and scalpel technique [Fig.5]. Antibiotics and analgesic were prescribed for the management of pain. After one week the pack was removed and the surgical area examined. The healing was uneventful and satisfactory [Fig.6a,6b]. No post-surgical complications were encountered. Patients were recalled on the $15^{\text {th }}$ day and $21^{\text {st }}$ day to observe healing. Observation for clinical reappearance of gingival melanin pigmentation and its intensity were done. The gingiva appeared pink and healthy with a firm consistency and completely devoid of inflammation and also free of any recurrent pigmentation. The patient was recalled after first and three months, gingiva was devoid of any inflammation or recurrent pigmentation [Fig.7,8].

\section{Discussion}

There are wide variations in gingival color in normal healthy persons. Degree of vascularization,
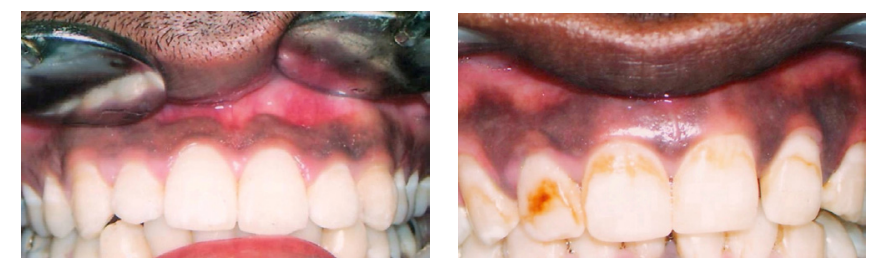

Fig.1(a): Pre-operative view of first patient. (b): Preoperative view of second patient.
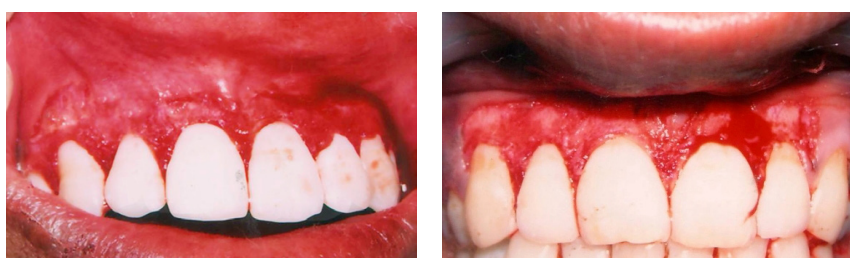

Fig.2(a): After removal of the hyperpigmented epithelium through scalpel technique in first patient. (b): Hyperpigmented epithelium removed in second patient.

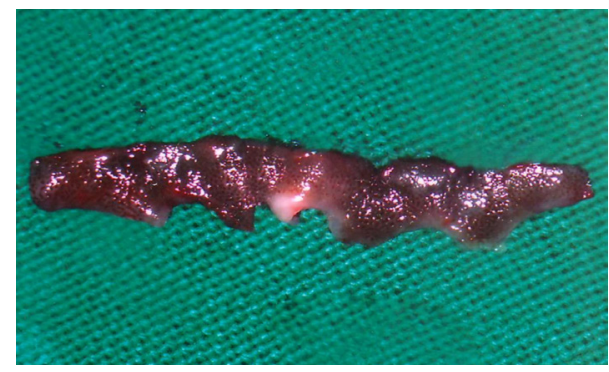

Fig.3: Hyperpigmented epithelium.

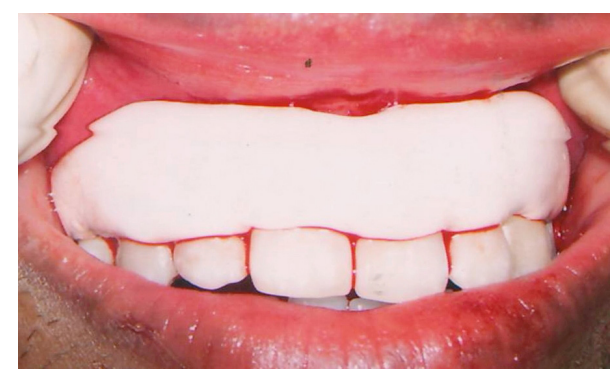

Fig.4: Periodontal dressing given.

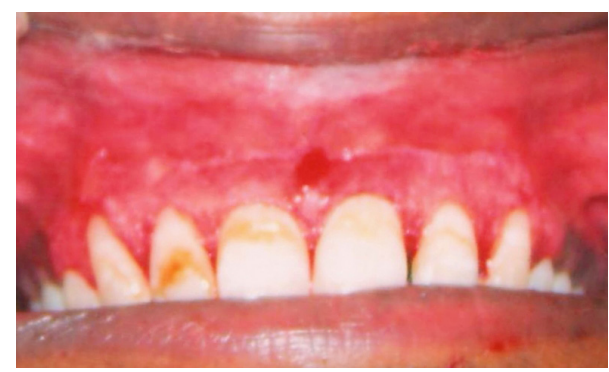

Fig.5: After scalpel and bur in second patient. 
keratinized layer thickness and the amount of the pigment containing cells generally determine the color of the gingiva [2]. The use of scalpel technique for the depigmentation is the most economical as compared to other techniques, which require more advanced armamentarium. However, scalpel surgery causes unpleasant bleeding during and after the operation, and it is necessary to cover the surgical site with periodontal dressing for 7 to 10 days $[5,6]$. Electrosurgery has its own limitations in that its repeated and prolonged use induces heat accumulation and undesired tissue destruction [5]. Cryosurgery is followed by considerable swelling and it is also accompanied by increased soft tissue destruction as the depth of penetration cannot be controlled [7].

Though the initial results of de-pigmentation procedure are highly encouraging, repigmentation is a common problem. The mechanism of repigmentation is not understood, but according to the migration theory, active melanocytes from the adjacent pigmented tissues migrate to the treated areas, causing repigmentation. Studies have shown that full clinical baseline repigmentation takes about 1.5-3 years. Variations may be due to different techniques performed or due to race of the patient. Thus this procedure is performed primarily for cosmetic reasons [8]. The use of scalpel technique for the depigmentation is the most economical as compared to other techniques, which require more advanced armamentarium. However, scalpel surgery causes unpleasant bleeding during and after the operation, and it is necessary to cover the surgical site with periodontal dressing for 7 to 10 days. Electrosurgery has its own limitations in that its repeated and prolonged use induces heat accumulation and undesired tissue destruction [9].

\section{Conclusion}

Depigmentation of hyperpigmented gingiva by scalpel surgery with bur abrasion is simple, easy to perform, cost effective and above all it causes less discomfort and is esthetically acceptable to the patient.
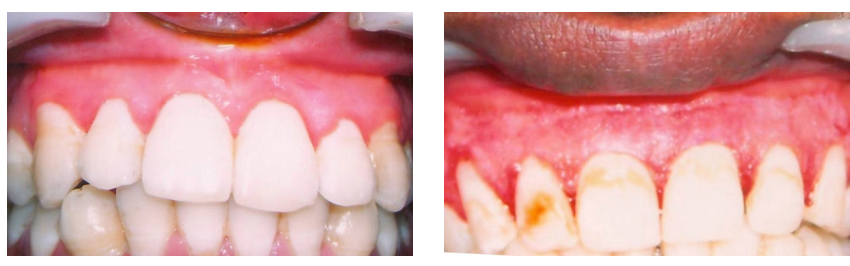

Fig:6(a): Post operative view in first patient. (b): Postoperative view in second patient.
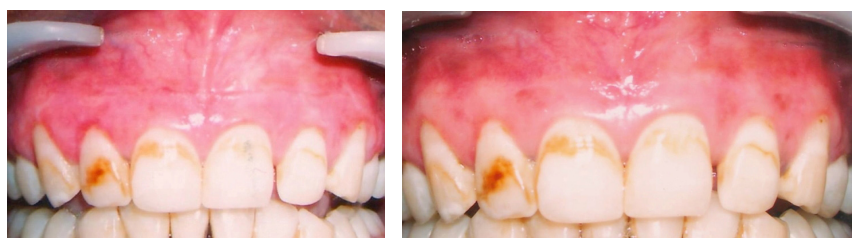

Fig:7: One month follow-up of second patient. Fig.8: Three months follow-up of second patient.

Contributors: JG. RM: case identification; JG, AG: performed procedure on both patients; RM, HM, HS: data collection and image editing. All authors contributed in writing and drafting manuscript Funding: None; Competing interests: None stated.

\section{References}

1. Dummett CO. Oral pigmentation:First symposium of oral pigmentation. J Periodontol. 1960;31:356-360.

2. Cicek Y, Ertas U. The normal and pathological pigmentation of oral mucous membrane: a review. J Contemp Dent Pract. 2003;4(3):76-86.

3. Deepak P, Sunil S, Mishra R, Sheshadri. Treatment of gingival pigmentation. A Case series. Indian J Dent Res. 2005;16:171-176.

4. Çerman AA, Altuna IK. Oral pigmentation. J Pigment Disorder. 2016;3:234.

5. Hirschfeld I, Hirschfeld L. Oral pigmentation and method of removing it. Oral Surg Oral Med Oral Path. 1951;4:1012

6. Gnanasekhar JD, al-Duwairi YS. Elecrosurgery in Dentistry. Quintessence Int. 1998;29:649-654.

7. Bishop K. Treatment of unslightly oral pigmentation: A case report. Dental Update. 1994;21(6):236-237.

8. Sushma L, Yogesh D, Marawarc PP. Management of gingival hyperpigmentation using surgical blade and diode laser therapy: A comparative study. J Oral Laser Appl. 2009;9:41-47.

9. Murthy MB, Kaur J, Das R. Treatment of gingival hyperpigmentation with rotary abrasive, scalpel, and laser techniques: A case series. J Indian Soc Periodontol. 2012;16(4):614-619. 